\title{
THE TREATMENT OF SINGULARITIES OF PARTIAL DIFFERENTIAL EQUATIONS BY RELAXATION METHODS*
}

\author{
BY \\ H. MOTZ \\ University of Sheffield, England
}

1. Introduction. In the course of a study of boundary value problems arising in radiation theory and electrostatics, the treatment of singularities demanded special attention. In most problems of practical importance boundaries with sharp corners occur. Such sharp corners give rise to singularities of various types. When the computed function is bounded, but has a branch point at the corner, the difficulty is not serious. The use of a graded net with a finer mesh size near the corner is possible. Conformal transformation which automatically provides a finer net near corners is also successful. The mesh size near the corner should be of the order of magnitude of the radius of curvature of the corner, and when this is small a mathematical idealization involving infinitely sharp corners is preferable. The special treatment outlined in this note makes use of such an idealization and shortens the labour considerably. Special treatment is essential when the function approaches infinite values near the corner.

2. Plane harmonic functions. Solutions of $\nabla^{2} \phi=0$ are bounded when the boundary condition prescribes constant values near the corners. It can be shown that they are also bounded when $\partial \phi / \partial \nu$ is constant, where $\nu$ is the direction normal to the boundary. This type of boundary condition occurs e.g. when two plane harmonic functions $\phi$ and $\psi$ are computed inside a boundary $B$ for the purpose of a conformal transformation

$$
x+i y=\phi(x, y)+i \psi(x, y)
$$

and $\psi=$ const. is specified at the boundary forming the corner. When expressed in polar coordinates $r, \vartheta$ centered at $P$ (Fig. 1), the equation

becomes

$$
\nabla^{2} \phi=0
$$

$$
r^{2} \frac{\partial^{2} \phi}{\partial r^{2}}+r \frac{\partial \phi}{\partial r}+\frac{\partial^{2} \phi}{\partial \vartheta^{2}}=0 .
$$

With $\phi(r, \vartheta)=R(r) \cdot \Theta(\vartheta)$, the following equations for $R$ and $\Theta$ are obtained

$$
\begin{aligned}
\frac{d^{2} \Theta}{d \vartheta^{2}}+n^{2} \Theta & =0, \\
r^{2} \frac{d^{2} R}{d r^{2}}+r \frac{d R}{d r}-n^{2} R & =0 .
\end{aligned}
$$

In these equations $n^{2}$ stands for a positive constant, and

$$
\frac{d \Theta}{d \vartheta}=0 \quad \text { when } \quad \vartheta=0, \quad \vartheta=\alpha .
$$

\footnotetext{
* Received Jan. 2, 1946.
} 
Hence

$$
\phi=\sum_{k=-\infty}^{\infty} A_{k} r^{n} \cos n \vartheta
$$

where $n=\pi k / \alpha(k=0, \pm 1, \pm 2, \pm 3, \cdots)$.

In order to investigate the terms with negative exponent in this series, we exclude the corner by a small circle of radius $\rho$. On this circle $(\partial \phi / \partial r)_{\rho \rightarrow 0}=0$. It is found that

$$
A_{-\varepsilon}=\rho^{2 n} A_{+o} \quad(s=1,2,3, \cdots) .
$$

When $\rho \rightarrow 0$, the circle contracts towards the point $P$ and the terms with negative exponents vanish. Thus $\phi$ will be represented by the series

$$
\phi=A_{0}+A_{1} r^{\pi / \alpha} \cos \frac{\pi}{\alpha} \vartheta+A_{2} r^{2 \pi / \alpha} \cos \frac{2 \pi}{\alpha} \vartheta+A_{3} r^{3 \pi / \alpha} \cos \frac{3 \pi}{\alpha} \vartheta+\cdots
$$

in the neighbourhood of $P$.

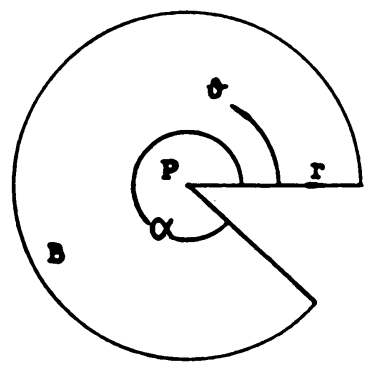

FIG. 1

3. Method of special treatment. The method of treatment will now be explained with reference to the example of a corner with $\alpha=2 \pi, \pi / \alpha=\frac{1}{2}$. In the treatment of two-dimensional problems by relaxation methods, ${ }^{1,2}$ the function $\phi$ is computed at points of a net with small but finite mesh size. Let us denote by $\phi_{0}$ the value of $\phi$ at such a point, by $\phi_{1}, \phi_{2}, \phi_{3}, \phi_{4}$ the values of $\phi$ at the nearest neighbouring points. The mesh length is $a$. At points where the function is regular, double Taylor expansion shows that

$$
a^{2} \nabla^{2} \phi-\sum_{m=1}^{4} \phi_{m}+4 \phi_{0}=-\frac{1}{12} \Delta_{0}^{i v}(x)-\frac{1}{12} \Delta_{0}^{i v}(y) \cdots,
$$

where $\Delta_{0}^{\mathrm{iv}}(x), \Delta_{0}^{\mathrm{iv}}(y)$, are the fourth central differences in the $x$ and $y$ direction, respectively, at the point where $\phi=\phi_{0}$. This expansion can only be used when the right hand side converges. At a singularity and its nearest neighbouring points this expansion is not valid. Figure 2 shows an example of a boundary where $\phi=0$ on $A E$, $\phi=1000$ on $E B$, and $\partial \phi / \partial \nu=0$ on all other boundaries. The Taylor expansion fails at

${ }^{1}$ H. W. Emmons, Numerical solution of partial differential equations, Quarterly Appl. Math., 2, 173-195 (1944).

${ }^{2}$ D. N. de G. Allen, D. G. Christopherson, L. Fox, J. R. Green, H. Motz, F. S. Shaw and R. V. Southwell, Relaxation methods applied to engineering problems, Phil. Trans. Royal Soc. London (A), 239, 367386, 419-537, 539-578 (1945). 
$P^{\prime}, Q^{\prime}, R^{\prime}$, and $S^{\prime}$. In order to obtain valid equations at these points, we consider series of the type (6) at the pivotal points $P, Q, R, S$

$$
\phi=A_{0}+A_{1} r^{1 / 2} \cos \frac{\vartheta}{2}+A_{2} r \cos \vartheta+A_{3} r^{3 / 2} \cos 3 / 2 \vartheta+\cdots .
$$

Only the first four terms are retained. The units of $r$ can be so chosen that $r=1$ at $P, Q, R, S$. In terms of $\phi_{P}, \phi_{Q}, \phi_{R}, \phi_{S}$ one obtains

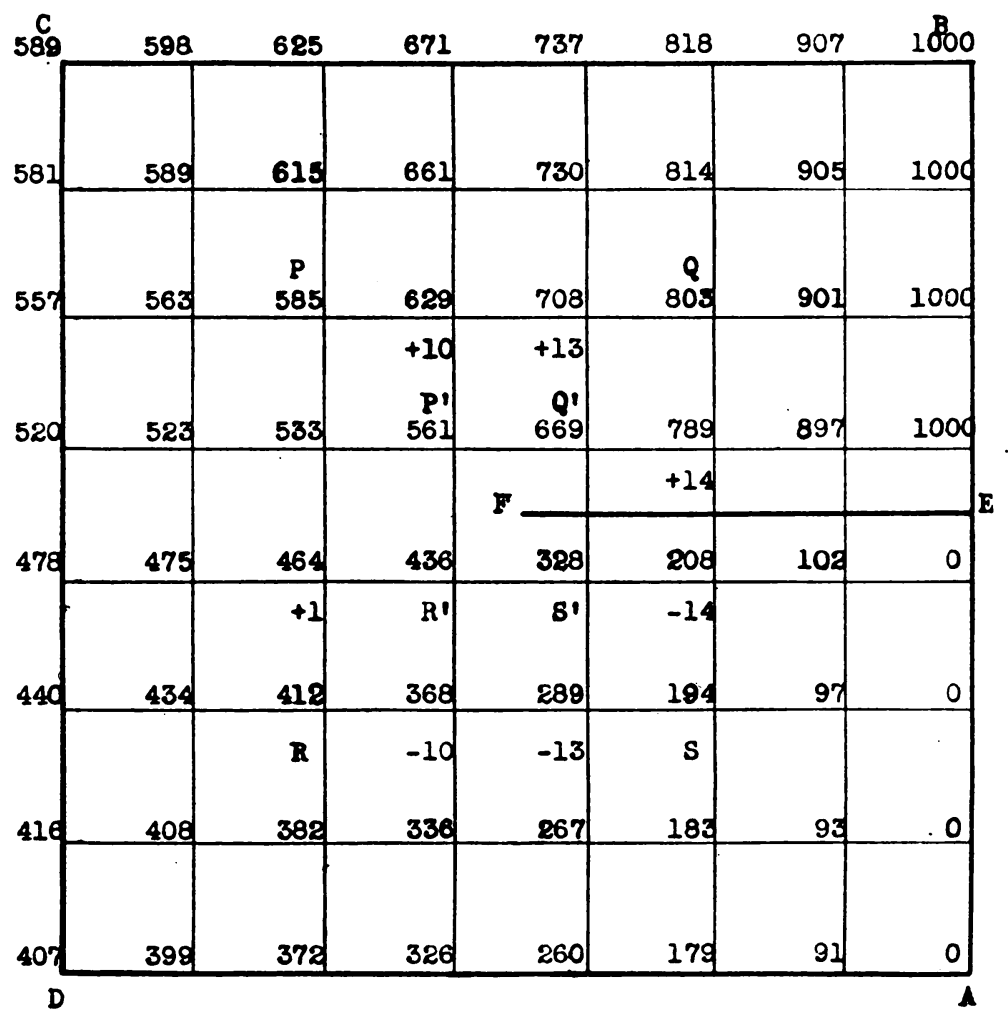

FIG. 2

$$
\begin{aligned}
& A_{0}=0.25\left(\phi_{P}+\phi_{Q}+\phi_{R}+\phi_{S}\right), \\
& A_{1}=0.191\left(\phi_{P}-\phi_{R}\right)-0.462\left(\phi_{S}-\phi_{Q}\right), \\
& A_{2}=0.354\left(\phi_{Q}-\phi_{P}-\phi_{R}+\phi_{S}\right), \\
& A_{3}=-0.191\left(\phi_{Q}-\phi_{S}\right)+0.462\left(\phi_{R}-\phi_{P}\right) .
\end{aligned}
$$

At the special points $P^{\prime}, Q^{\prime}, R^{\prime}$, and $S^{\prime}$, we find from (8)

$$
\begin{aligned}
& \phi_{P^{\prime}}=0.457 \phi_{P}+0.235 \phi_{Q}+0.209 \phi_{R}+0.099 \phi_{S}, \\
& \phi_{Q^{\prime}}=0.235 \phi_{P}+0.593 \phi_{Q}+0.099 \phi_{R}+0.073 \phi_{S}, \\
& \phi_{R^{\prime}}=0.209 \phi_{P}+0.099 \phi_{Q}+0.457 \phi_{R}+0.235 \phi_{S}, \\
& \phi_{S^{\prime}}=0.099 \phi_{P}+0.073 \phi_{Q}+0.235 \phi_{R}+0.593 \phi_{S} .
\end{aligned}
$$


These are the equations used at special points. The relaxation procedure is carried out normally everywhere, observing that equations (10) hold at special points. The residual at a special point due to an increment at a pivotal point $P$ is therefore the product of this increment with the coefficient of $\phi_{P}$ in the equation which holds at the special point. This is in accordance with the usual relaxation procedure. The removal of a residual at a special point is particularly easy. It is simply subtracted from the value of $\phi$ at the special point in question. Due to this removal the usual residuals accrue at ordinary neighbouring points, but of course, no residuals are passed on to special points.

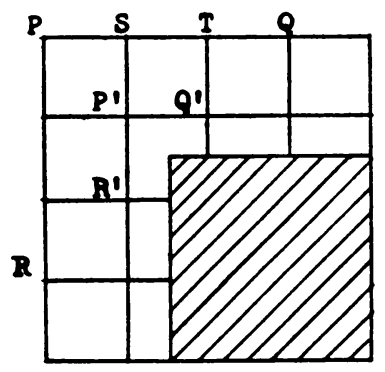

Fig. 3

Figure 3 refers to an example with $\alpha=3 \cdot \pi / 2$. Here we retain three terms only Special points are $P^{\prime}, Q^{\prime}, R^{\prime}$, pivotal points $P, Q, R$. The equations at special points are

$$
\begin{aligned}
& \phi_{P^{\prime}}=0.486 \phi_{P}+0.257 \phi_{Q}+0.257 \phi_{S}, \\
& \phi_{Q^{\prime}}=0.257 \phi_{P}+0.612 \phi_{Q}+0.131 \phi_{S}, \\
& \phi_{R^{\prime}}=0.257 \phi_{P}+0.131 \phi_{Q}+0.612 \phi_{S} .
\end{aligned}
$$

It should be checked whether the three first terms of the series

$$
\phi=A_{0}+A_{1} r^{2 / 3} \cos \frac{2}{3} \vartheta+A r_{2}^{4 / 3} \cos \frac{4}{3} \vartheta+\cdots
$$

which has been used for the derivation of (11) represent the function $\phi$ adequately. This is done by comparing the result of the relaxation computation at points $S, T$, where ordinary difference equations have been used with the values of $\phi$ calculated by means of the first three terms of (12).

A similar check was carried out at analogous points in the example of Fig. 2. It was found that the agreement was not satisfactory. The errors have been recorded in Fig. 2 underneath the respective $\phi$ values. In this case it is possible (with the net shown in Fig. 4) to retain five terms of the series. Pivotal points are $T, U, V, W, X$; special points $T^{\prime}, U^{\prime}, V^{\prime}, W^{\prime}, X^{\prime}$, and the equations for $\phi$ at special points are

$$
\begin{aligned}
& \phi_{T^{\prime}}=0.546 \phi_{T}+0.313 \phi_{U}+0.062_{5} \phi_{V}+0.062_{5} \phi_{W}+0.016 \phi_{X}, \\
& \phi_{U^{\prime}}=0.156 \phi_{T}+0.578 \phi_{U}+0.188 \phi_{V}+0.047 \phi_{W}+0.031 \phi_{X}, \\
& \phi_{V^{\prime}}=0.031 \phi_{T}+0.188 \phi_{U}+0.562 \phi_{V}+0.188 \phi_{W}+0.031 \phi_{X}, \\
& \phi_{W^{\prime}}=0.031 \phi_{T}+0.047 \phi_{U}+0.188 \phi_{V}+0.578 . \phi_{W}+0.156 \phi_{X}, \\
& \phi_{X^{\prime}}=0.016 \phi_{T}+0.062_{5} \phi_{U}+0.062 \phi_{V}+0.313 \phi_{W}+0.546 \phi_{X} .
\end{aligned}
$$


The result is again checked by comparing the result of the relaxation procedure with the $\phi$ values near the corner calculated from

$$
\phi=A_{0}+A_{1} r^{1 / 2} \cos \frac{1}{2} \vartheta+A_{2} r \cos \vartheta+A_{3} r^{3 / 2} \cos \frac{1}{2} \vartheta+A_{4} r^{2} \cos 2 \vartheta,
$$

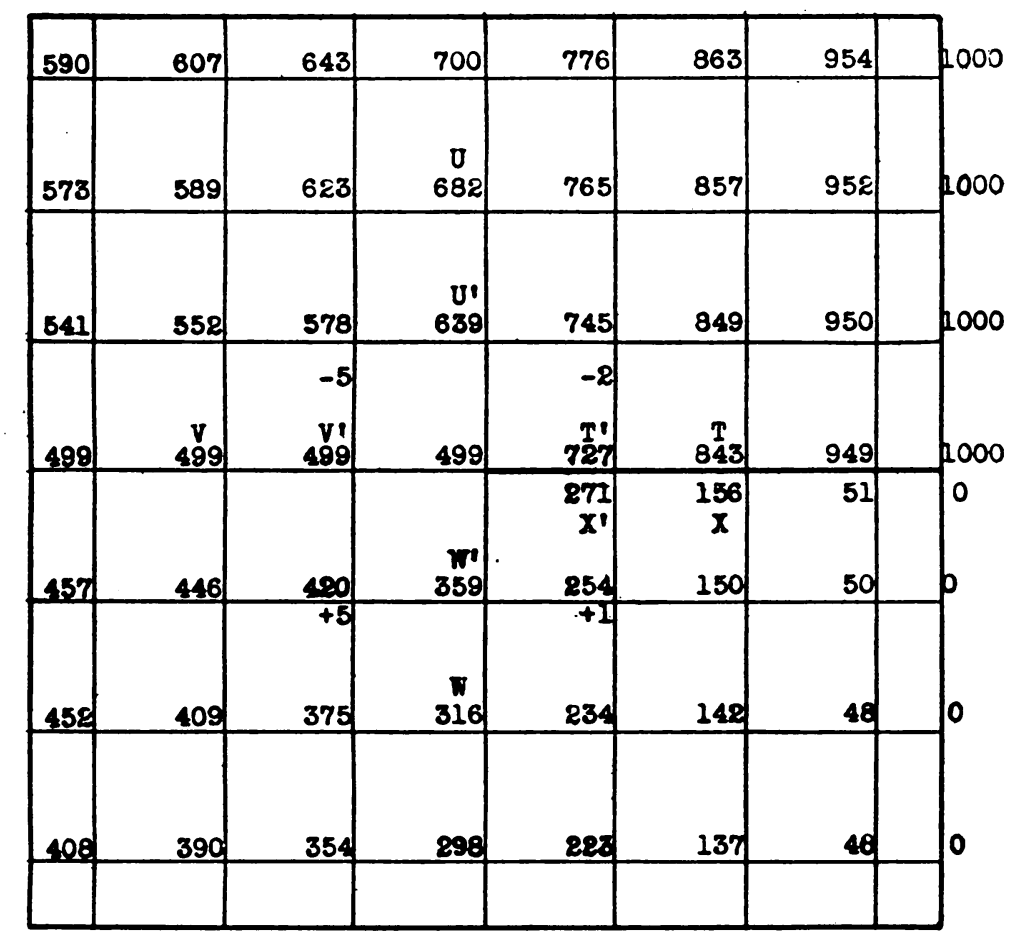

Fig. 4

where the $A$ 's are given by

$$
\begin{aligned}
& A_{0}=0.250\left(\phi_{U}+\phi_{V}+\phi_{W}\right)+0.125\left(\phi_{T}+\phi_{X}\right), \\
& A_{1}=0.354\left(\phi_{U}-\phi_{W}\right)+0.250\left(\phi_{T}-\phi_{X}\right), \\
& A_{2}=-0.500 \phi_{V}+0.250\left(\phi_{T}+\phi_{X}\right), \\
& A_{3}=0.354\left(\phi_{W}-\phi_{U}\right)+0.250\left(\phi_{T}-\phi_{X}\right), \\
& A_{4}=0.250\left(\phi_{V}-\phi_{U}-\phi_{W}\right)+0.125\left(\phi_{T}+\phi_{X}\right) .
\end{aligned}
$$

The agreement is now much better. In Fig. 4 the errors have been recorded. It will be noticed that the mesh points of Fig. 2 lie between those of Fig. 4. By interpolating values at midpoints of the meshes of Fig. 4, we find that the solutions given in the two figures are in fair agreement.

When the above test fails, a finer net should be used as a rule, because the calculation becomes rather cumbersome when more than five terms of the series are retained.

To obtain, without the special treatment, a result which differs from the one of Fig. 2 by less than $1 \%$ at any point of the net, the net near the corner would have to be 7 times as fine. 
4. Other examples. As an example of a corner where the value of the function is specified, let us consider an electrostatic potential $\psi$. In this case the series is

$$
\psi=A_{0}+A_{1} r^{1 / 2} \sin \frac{1}{2} \vartheta+A_{2} r \sin \vartheta+A r^{3 / 2} \sin \frac{3}{2} \vartheta+\cdots .
$$

The components of the electric field in Cartesian cordinates, $E_{x}, E_{y}$, are not bounded on a sharp corner when $\alpha>\pi$. Let us consider the term $r^{n} \cos n \vartheta$ of the series (5). $E_{x}$ and $E_{y}$ will contain terms $r^{n-1} \sin (n-1) \vartheta, r^{n-1} \cos (n-1) \vartheta$, respectively, and negative exponents of $r$ will therefore occur when $\alpha>\pi$. The method outlined above can still be used to compute a function with such a singularity. In the case $\alpha=2 \pi$ the negative exponent $-\frac{1}{2}$ occurs. Terms with exponents $-\frac{1}{2}$ and $+\frac{1}{2}$ depend on $\vartheta$ in the same manner. It is therefore necessary to choose pivotal points which have not all the same distance from the corner.

The method is equally applicable to solutions of the wave equation

$$
\nabla^{2} \phi+k^{2} \phi=0 .
$$

In Cartesian coordinates and with $\xi=x / a, \eta=y / a$ (where $a$ is the mesh length of the net), Eq. (16) becomes

$$
\frac{\partial^{2} \phi}{\partial \xi^{2}}+\frac{\partial^{2} \phi}{\partial \eta^{2}}+k^{2} a^{2} \phi=0
$$

Referring again to the case $\alpha=2 \pi, \partial \phi / \partial \nu=0$ and retaining five terms, we see that the expression (5) holds at pivotal points. At the special points we have

$$
\begin{aligned}
\phi=A_{0} & +A_{1} \frac{J_{1 / 2}(k a)}{J_{1 / 2}(2 k a)} \cos \frac{1}{2} \vartheta+A_{2} \frac{J_{1}(k a)}{J_{1}(2 k a)} \cos \vartheta+A_{3} \frac{J_{3 / 2}(k a)}{J_{3 / 2}(2 k a)} \cos \frac{8}{2} \vartheta \\
& +A_{4} \frac{J_{2}(k a)}{J_{2}(2 k a)} \cos 2 \vartheta,
\end{aligned}
$$

where $J_{n}$ are the Bessel functions of order $n$. When $k a<0 \cdot 1$, the ratios $J_{n}(k a) / J_{n}(2 k a)$ differ from $\left(\frac{1}{2}\right)^{n}$ only in the third decimal. When the mesh length $a$ is small compared with the wave length $l=2 \pi / k$, the special equations are therefore the same for solutions of the wave equation and those of Laplace's equation.

5. Conformal transformation. When a solution of more complicated differential equations, e.g. the equations of viscous flow, or $\nabla^{4} F=0$, is computed it is of ten an advantage to remove singularities at the boundary by a conformal transformation $\phi=\phi(x, y), \psi=\psi(x, y)$. Let us suppose that it is desired to transform the interior of the boundary shrwn in Fig. 2 into the interior of a rectangle in the $\phi, \psi$ plane.

The lines $\phi=$ const. at suitable intervals can be found from the $\phi$-values recorded in Fig. 2. The lines $\psi^{\prime}=$ const. are orthogonal to the lines $\phi=$ const. and are best computed separately.

The condition for orthogonality

$$
\frac{\partial \phi}{\partial x} \frac{\partial \psi^{\prime}}{\partial x}+\frac{\partial \phi}{\partial y} \frac{\partial \psi^{\prime}}{\partial y}
$$

is satisfied when 


$$
\frac{\partial \phi}{\partial x}=\lambda \frac{\partial \psi^{\prime}}{\partial y}, \quad \frac{\partial \phi}{\partial y}=-\lambda \frac{\partial \psi^{\prime}}{\partial x}
$$

where $\lambda$ is a constant. From these equations it follows that

$$
\frac{\partial^{2} \phi}{\partial x^{2}}+\frac{\partial^{2} \phi}{\partial y^{2}}=0, \quad \frac{\partial^{2} \psi^{\prime}}{\partial x^{2}}+\frac{\partial^{2} \psi^{\prime}}{\partial y^{2}}=0 .
$$

The boundary conditions for $\psi^{\prime}$ are $\partial \psi^{\prime} / \partial \nu=0$ on $E A, E B, \psi^{\prime}=0$ on $E F, \psi^{\prime}=$ const. on $A D, D C$, and $C B$. The last constant is arbitrary and may be given a convenient value, e.g. 1000 for three figure accuracy.

It is easily seen that it is necessary to determine the constant $\lambda$ in (18), in order to carry on with the computation of the original equation (e.g. $\nabla^{4} F=0$ ). In the coordinates $\phi$ and $\psi=\lambda \psi^{\prime}$, this equation becomes

$$
\left(\frac{\partial^{2}}{\partial \phi^{2}}+\frac{\partial^{2}}{\partial \psi^{2}}\right)\left(\frac{\partial^{2}}{\partial \phi^{2}}+\frac{\partial^{2}}{\partial \psi^{2}}\right) F=0
$$

The constant $\lambda$ is determined by (18). These equations can be regarded as an estimate of $\lambda$ at every point. Denoting the finite differences in the $x$ and $y$ directions at the mesh point $i$ by

$$
D_{x} \phi(i), \quad D_{y} \phi(i), \quad D_{x} \psi^{\prime}(i), \quad D_{y} \psi^{\prime}(i),
$$

the quantities $\delta_{1}(i)$ and $\delta_{2}(i)$ defined by

$$
\delta_{1}(i)=D_{x} \phi(i)-\lambda D_{y} \psi^{\prime}(i), \quad \delta_{2}(i)=D_{y} \phi(i)+\lambda D_{x} \psi^{\prime}(i)
$$

are not all zero, but constitute a measure of the computational error. It is desired to find a mean value for $\lambda$ for which the variance of the computational error is a minimum. The sum

$$
\sum_{i}\left[\delta_{1}^{2}(i)+\delta_{2}^{2}(i)\right]
$$

is thus minimized with respect to $\lambda$ and the following expression for $\lambda$ is obtained:

$$
\lambda=\frac{\sum_{i}\left\{D_{x} \phi(i) D_{y} \psi^{\prime}(i)-D_{y} \phi(i) D_{x} \psi^{\prime}(i)\right\}}{\sum_{i}\left(\left\{D_{x} \psi^{\prime}(i)\right\}^{2}+\left\{D_{y} \psi^{\prime}(i)\right\}^{2}\right)} .
$$

It has been found that, with the help of this technique of separate computation of the two transformation functions and using the special treatment of corners at the boundary conformal transformations can be computed with great accuracy.

6. Acknowledgment. The numerical work for this paper was done by Miss L. Klanfer whose services were put at the author's disposal by the Directorate of Scientific Research of the British Admiralty. 